\title{
Ueber die Entstehung des sogenannten angeborenen muskulären Schiefhalses.
}

\author{
Von \\ Dr. Oscar Witzel, \\ Professor der Chirurgie in Bonn.
}

In einem jedem Theile der Natur - und Heilkunde giebt es einzelne Lehren, die so einfach und einleuchtend erscheinen, dass sie, einmal aufgestellt, ohne Anstand immer weiter übernommen werden; eine erneute Beweisführung wird für überflüssig, eine Anzweifelung für unberechtigt gehalten. Dennoch ist mancher, anscheinend für alle Zeiten feststehender Satz arg ins Wanken gerathen, wenn in streng kritischer Weise der Grund untersucht wurde, auf dem er aufgefïhrt war, das Material besichtigt wurde, das zu seinem Aufbau diente.

So galt für die Entstehung des angeborenen muskulären Schiefhalses bis vor kurzem fast unbestritten die Lehre, nach welcher das Uebel auf ein, während der Geburt den kindlichen Hals treffendes, Trauma zurückzuführen ist. Von Stromeyer zuerst aufgestellt, war der Satz bald in Lehrbücher und Vorträge übergegangen und darin verblieben. Die Nachforschung in Fällen von schiefer, seit der frühesten Kindheit bestehender Kopfstellung, bei denen sich eine strangartige Umwandlung und Verkürzung eines Kopfnickers fand, ergab den Chirurgen, dass ungemein häufig Störungen bei der Geburt vorhanden gewesen waren, sofern überhaupt in dieser Hinsicht sich noch etwas feststellen liess. - Da erschien vor sechs Jahren im 30. Bande von Langenbeck's Archiv für klinische Chirurgie die Arbeit Petersen's: Caput obstipum. (Zur Aetiologie und Behandlung.)

Petersen machte in seiner Mittheilung darauf aufmerksam, dass wir bis da zu Unrecht die Kette der Beweisführung als geschlossen betrachteten. Bei aufmerksamer Verfolgung seiner Darstellung wird man ohne Weiterung zugeben, dass wirklich in der Literatur nach S tr o m e y er nicht genügend gestützte Behauptungen 
Witzel, Ueber die Entstehg. des sog. angeb. muskul. Schiefhalses. 125

als bewiesen weiter geführt worden sind. Wenn aber Petersen zu dem Senlusse kam, dass , der Riss des Kopfnickers in der Aetiologie des Torticollis zu streichen ist", so musste dies doch höchlichst befremden. Der verstorbene Maas sprach deshalb wohl im Sinne der grossen Mehrzahl seiner Fachgenossen, als er einem Berichte über die Arbeit (Fortschritte der Medicin, 15. Nov. 1884) die Bemerkung hinzufügte: „Die Entstehung der Caput obstipum durch Läsion des Musculus sternocleido-mastoideus bei der Geburt steht durch viele gute Beobachtungen so fest, dass Petersen hieran nichts ändern wird."

Damit konnte jedoch selbstredend die Sache nicht als abgethan erachtet werden; es galt bessere Stützen für Stromeyer's Lehre zu schaffen, wenn anders dieselbe nicht doch fallen sollte. - An der wissenschaftlichen Entwickelung der Frage war ich schon einigermaassen betheiligt durch anatomische und experimentelle Untersuchungen, die von mir vor nunmehr einem Jahrzehnt angestellt wurden, und deren Ergebniss sich findet in den Beiträgen zur Kenntniss der secundären Veränderungen beim muskulären Schiefhalse (Deutsche Zeitschrift für Chirurgie, Bd. XVIII). Im März des Jahres 1885 wurden dann nach dem Erscheinen der Veröffentlichung Petersen's vorläufig die an unserer Klinik gemachten Erfahrungen in einer Dissertation unseres Schülers B. Fabry zusammengefasst. Mit guter Berechtigung konnte in der Arbeit für die alte Theorie eingetreten werden. Die folgenden sechs Jahre haben uns dann reichlich Gelegenheit geboten, weitere Beobachtungen zu machen und eine Zahl von Fällen zusammenzubringen, welche auch eine statistische Schlussfolgerung zulässt. Vom Thierversuche haben wir hier, wie auch Fabry nach mir erfuhr, keine weiteren Aufschlüsse zu erwarten. Zu vermuthen ist, dass auch Petersen bei seinen beabsichtigten Versuchen nicht mehr Glück gehabt hat; es fehlt eben das geeignete Thier, welches die Haltung des Menschen hat. Dagegen erscheint mir durch unsere und fremde weitere Erfahrungen die Sache klinisch dem Spruche so nahe gebracht, als das von chirurgischer Seite geschehen kann. Die weitere Förderung der Frage kann nur von Seiten der Geburtshelfer erwartet werden, vor deren Forum sie hiermit gestellt sei. - Eine Wiedergabe auch dessen, was die frühere chirurgische Literatur an Bemerkenswerthem bietet, erschien zweckmässig bei dieser Uebertragung. Nicht Jedem sind die Originalien Stromeyer's, Dieffenbach's u. s. w. leicht zugängig. Wo es sich um grundsätzliche Verschiedenheiten der 
Anschauung handelt, wurde wörtlich angeführt, um die Freiheit des Urtheils auch nicht durch Wortfassung zu beeinflussen.

Das in Rede stehende Leiden ist zweifellos zu allen Zeiten beobachtet worden. Alexander der Grosse litt nach den Untersuchungen von $\mathrm{Dechambr} \mathrm{e}^{1}$ ), an einem Schiefhalse geringen Grades mit Neigung nach rechts und mit leichter Schädelskoliose, wenn die im Louvre befindliche, als Hermes Alexandre bezeichnete Büste wirklich eine Copie des von Lysippus gemeisselten Bildnisses ist. - Römische Classiker, Suetonius und Horaz, erwähnen die Cervix obstipa und das Caput obstipum.

In der medicinischen Literatur handeln zuerst vom Schiefhalse vor 200 Jahren Nicolaus Tulpius²) und Job van Meekren ${ }^{3}$ ), welche gleich richtig den Sitz des Uebels in den Kopfnicker verlegen. H. von Roonhuysen ${ }^{4}$ ) ist der Erste, welcher unter Beschreibung zweier Fälle klar ausspricht, dass die Erkrankung angeboren sei. „Ein Junger gesell von 23 Jahren konnte von Mutterleib an seinen Kopf nicht gen Himmel aufrichten, als der mit einem übernatürlich verhärteten, eingekrümmten und eingeschrumpfenen Bande als eine Senne, am Ende und dem Mittel des Schlüsselbeines vestgemacht: wordurch der Kopf vermittelst der, mit denen düttenförmigen Vorgängen des Hinterhauptes habenden vesten Vereinigung nach der linken Schulter gezogen und gehalten wurde." Der zweite Fall betrifft „eines aufm Russland wohnenden Mannes, Johann Roelof's Sohn, von 16 Jahren, so einen krummen Hals mit zur Welt gebracht und zwar auf eine solche Art, als in vorher-

1) H. Bouvier, Leçons cliniques sur les maladies chroniques de l'appareil locomoteur. 1858. p. 88, 89. - Bei meiner Anwesenheit in Paris hatte ich jüngst Gelegenheit, die von Dechambre gemachten Angaben zu prüfen. Die betreffende Portrait-Büste findet sich in der Salle de la Pallas de Velletri, IX, 1763; sie bietet durchaus die Veränderungen eines alten Caput obstipum musculare, und zwar eines solchen, welches in Behandlung gestanden hat. Der Kopf ist bei entsprechender Verkürzung der rechten Halsseite nach rechts geneigt und ein wenig nach links gedreht. Das Gesicht weist die typische Skoliose mit Convexität nach links hin auf. Die rechte Gesichtshalfte ist niedriger und etwas abgeplattet, die linke höher und am Uebergange von der mittleren zur seitlichen Partie schärfer abgebogen. Die Entfernung des Mundwinkels vom äusseren Augenlidwinkel misst rechts $6,6 \mathrm{~cm}$, links $7,4 \mathrm{~cm}$.

2) Observ. med. Lib IV, cap. 58.

3) Heel-en Geneeskonstige anmerkkingen, cap. 30, p. 172.

4) "Historischer Heilcuren in zwey Theile verfassete Anmerkungen." S. 82 ff. 
gehender Anmerkung beschrieben ist; jedoch mit diesem Unterscheid, dass die Senne nicht allein viel dicker und grösser, sondern bey den Schlüsselbein entzwey gespalten war."“

Die Wundärzte des vorigen Jahrhunderts, die in den ersten Decennien des unserigen wirkenden und schreibenden, verfolgten mehr die praktische Frage der Heilung uud nahmen ohne viel Theorie den Zustand als angeboren hin; sie erwähnen wohl auch gar nicht, dass es sich um eine angeborene Störung handelt, so Dieffenbach nach 1830 in Rust's Handbuch der Chirurgie. In dem Artikel über Caput obstipum heisst der bezügliche Satz auf Seite 625: ,Nicht selten sehen wir auch den schiefen Hals durch eigenthümliche Verhärtungen, welche sich in der Substanz des Muskels ausbilden, entstehen; ich habe drei Fälle der Art, und zwar alle bei kleinen Kindern, beobachtet und alle drei durch innere und äussere Mittel vollkommen geheilt."

Dem gegenüber bietet die Darstellung, welche acht Jahre später Louis Stromeyer, der geniale Begründer der operativen Orthopädie, in seiner klassischen Monographie ${ }^{1}$ ) gegeben hat, neue grosse Gesichtspunkte. Stromeyer ist so oft falsch verstanden worden, dass es geboten erscheint, den von der Entstehung des Schiefhalses handelnden Theil wörtlich zu geben: „Ein sehr merkwürdiger und, so viel ich weiss, bis jetzt nicht beachteter Umstand ist das Zusammentreffen angeborener Verkürzung des Kopfnickers mit unregelmässiger Kindeslage, so dass eine Steissgeburt eintrat oder die Wendung erforderlich wurde. Die nachfolgenden Operationsgeschichten liefern dazu einige Beiträge, und es sind mir durch Freunde mehrere ähnliche Fälle bekannt geworden, wo Kinder mit Caput obstipum durch die Zange oder durch die Wendung unter grossen Schwierigkeiten zur Welt befördert waren. Es sind hier verschiedene Fälle denkbar. Es kann zuerst schon durch die Lage im Uterus zur Entstehung des schiefen Halses die Veranlassung gegeben werden. Ich bin weit entfernt, zu glauben, dass die Lage allein schon ein solches Uebel zu erzeugen im Stande sei, aber wenn das Kind im Mutterleibe zu Krämpfen geneigt ist, so können diese ihre Richtung dadurch auf den Kopfnicker nehmen, wenn eine Seite des Halses erschlafft ist."

1) Louis Stromeyer, Beiträge zur operativen Orthopädik oder Erfahrungen über die subcutane Durchschneidung verkürzter Muskeln und deren Sehnen. Hannover 1838. 
„Es kann aber auch zweitens durch den Act der Geburt erst die Veranlassung zu Caput obstipum gegeben werden, entweder durch eine unvorsichtige Anwendung der Zange, oder durcl Zerren an dem vor dem Kopfe geborenen Rumpfe, wie dies von unwissenden Hebammen wohl häufig genug geschieht, wie in Nr. XLVII und IL. In diesem Falle wird die Schiefheit des Halses erst später bemerkt werden. Es entsteht hier die Frage: ob die ausgeübte Gewalt den Muskel selbst oder die obersten Halswirbel betreffe? welche sich vielleicht infolge derselben entzünden und einen Reflex auf die Halsmuskeln erzeugen. Einige Geburtshelfer, welche ich über diesen Punkt befragte, waren mehr geneigt zu glauben, dass die Gewalt die Halswirbel treffen müsse, aus Gründen, welche hier $\mathrm{zu}$ entwickeln $\mathrm{zu}$ weitläufig sein würde. Ich glaube indess, dass jedenfalls auch der andere Fall vorkommt; denn im Jahre 1837 habe ich eine offenbare Zerreissung des Kopfnickers linker Seite nach einer sehr rasch von einem geschickten Geburtshelfer beendeten Zangengeburt gesehen. Ich wurde drei Wochen nach der Geburt hinzugerufen und fand eine callöse Geschwulst an dem Kopfnicker dicht über seiner Sehne, welche sich ganz und gar so anfühlte, wio die nach der Durchschneidung des Muskels sich bildende Geschwulst, die erst nach einigen Wochen sich zu zertheilen pflegt. Die Zertheilung kam. auch hier, bis auf eine kleine harte Narbe, welche die dagewesene Verletzung um so deutlicher erkennen liess, zu Stande. Das Kind starb im dritten Monate an Lungenlähmung ganz plötzlich, nachdem es Tags vorher mit mehreren anderen Mitgliedern der Familie von einem Katarrhalfieber befallen worden war. $\mathrm{Zu}$ meinem unsäglichen Verdrusse wurde mir die Section nicht gestattet. Auch Dieffenbach in seinem Artikel Caput obstipum spricht von Geschwülsten in der Substanz des Kopfnickers bei kleinen Kindern, die sich leicht zertheilen liessen. - Wenn übrigens der Kopfnicker bei einer Zangengeburt abreissen kann, so kann er auch solche Zerrungen erleiden, welche eine habituelle Contractur hinterlassen."

,Dieser Gegenstand wird sehr leicht ins Reine gebracht werden, wenn sich die Wundärzte in jedem Falle von Caput obstipum erkundigen, auf welche Weise die Geburt stattgefunden hat.“

Es muss nun Petersen durchaus darin Recht gegeben werden, dass Stromeyer seine Theorie nicht in einer Weise durch Mittheilung entsprechender Beobachtungen gestützt hat, welche über- 
zeugend wirken konnte. Wir finden bei Stromeyer nur 4 Fälle erwähnt, und zwar XLVII zwei Geschwister, die in Steisslage geboren und dabei von einer etwas gewaltthätigen Hebamme ausgezogen wurden; unter XLVIII der Fall eines Mädchens, welches natürlich in Steisslage zur Welt kam und gleich den Kopf schief hielt; IL ist ein Fall in dem ein ebenfalls von der Hebamme gewendetes und unter bedeutendem Ziehen am Rumpfe entwickeltes Kind eine Schiefheit zeigte, als es den Kopf zu tragen anfing.

Vielfach wird nun Dieffenbach als Vertreter der von Stromeyer aufgestellten Ansicht genannt; aber mit Unrecht. Wohl war Dieffenbach ein begeisterter Bewunderer und Nachahmer der von Jenem geübten Methode subcutaner Operationen; aber nur für seltenere Fälle giebt er in seiner späteren Monographie $^{1}$ ) zu, dass die Schiefheit des Kopfes Folge des Geburtsherganges sein könne.

„Der schiefe Hals, welcher seinen Grund in einer Verkürzung des einen M. sterno-cleido-mastoideus hat, ist entweder wirklich angeboren, oder er ist durch die Geburt entstanden, oder er hat sich später entwickelt. Am häufigsten ist derselbe wohl schon früh im Mutterleibe vorhanden und weit seltener Folge einer schweren Geburt. Wenn ich unter einigen vierzig an Caput obstipum Operirten nur ein Viertheil der Zahl mit Verkürzung des linken Sterno-cleido-mastoideus sah, so möchte dies wohl für meine Meinung sprechen; denn die schwere Geburt mit der Zange oder durch die Wendung würde ebenso oft einen nachtheiligen Einfluss auf den linken als auch auf den rechten Kopfnicker haben." Vielmehr scheint nun Dieffenbach geneigt, ein intrauterines Zustandekommen der Verkürzung des Kopfnickers anzunehmen; jedenfalls ist er derjenige Forscher, welcher zuerst (S. 22) näher angiebt, wie man sich dasselbe denken könne.

„Wenn bei einem wenig geneigten Becken der Kopf mehrere Monate vor der gewöhnlichen Zeit in das Becken hinabtritt und sich als Caput ponderosum feststellt, so bleibt er während dieser ganzen Zeit in derselben seitlichen Stellung. Da nun der Erfahrung gemäss unter hundert Kopflagen siebzig Mal die erste Kopflage vorkommt, so mag hierin der Grund liegen, weshalb die angeborene Obstipitas colli viel häufiger auf der rechten, als auf

1) J. F. Dieffenbach, Ueber die Durchschneidung der Sehnen und Maskeln. Berlin 1841. 
der linken Seite rorkommt. Diese Mittheilung verdanke ich Herrn $\mathrm{Busch}$, welcher einem Vortrage von mir über den schiefen Hals in der $\mathrm{Hufel}$ and'schen medicinischen Gesellschaft beiwohnte."

In der Folgezeit geben dann. die Autoren der traumatischen Genese bald geringeren Raum, wie dies besonders in französischen Bearbeitungen des Gegenstandes geschieht, bald einen grösseren. So lassen G. F is cher (Deutsche Chirurgie, Lfg, 34), ferner Billroth und Winiwarter in ihrem Lehrbuche den Ursprung in utero neben einem solchen durch Verletzung bei der Geburt zu. Als höchst bemerkenswerth heben wir ferner hervor, dass einer der besten Kenner der einschlägigen Verhältnisse, Volkman n, in Pitha-Billroth's Handbuch ein jedes Caput obstipum musculare als durch Kopfnickerriss bei der Geburt bedingt darstellte. Dies Eintreten Volkmann's für Stromeyer's Ansicht trug zweifellos in ganz besonderem Maasse dazu bei, die letztere bei uns zur herrschenden zu machen.

Da erschien nun 1884 die Arbeit Petersen's, welche die inzwischen eingebürgerte Lehre als irrig darzuthun bezweckte, ohne indess zum eigenen Bedauern des Verfassers etwas Nenes dafür geben zu können. Petersen giebt zu, dass in einer Reihe von Fällen nach der Geburt Schwellungen im Bereiche eines Kopfnickers zur Beobachtung kommen, die man als Folge eines in der Geburt entstandenen Risses des Muskels auffassen kann; nach Bohn's Darstellung sei diese Kopfnickergeschwulst gelegentlich Veranlassung zu vorübergehender Schiefheit des Halses gewesen; es sei aber in der Literatur kein einziger Fall beschrieben, aus dem sicher erwiesen wäre, dass ein bei der Geburt am vorher unveränderten Kopfnicker entstandener Riss ein bleibendes Caput obstipum zur Folge hatte. Wenn man ein Hämatom eines Kopfnickers und ein bleibendes Caput obstipum an einem Kinde nachweisen würde, dann sei es doch von vornherein viel wahrscheinlicher, dass der Torticollis die prädisponirende Ursache der Zerreissung, nicht aber die Folge ist. Aus Mangel an Besserem geht Petersen schliesslich auf den Einfluss der Erblichkeit zurück. Wir werden diesen Punkt später näher würdigen. Es mag hier vorläufig nur erwähnt sein, dass Volkmann bald nach Petersen's Veröffentlichung (Centralbl. f. Chir. 1885, Nr. 14) Gelegenheit nahm, seine abweichende Meinung zu äussern.

Ein Erklärungsversuch für das intrauterine Entstehen der Kopfnickerverkürzung ähnlich dem von Busch geäusserten, nur 
bei umgedrehtem, in Steisslage versetztem Kinde, ist in jüngster Zeit ron M. Schmidt gemacht worden. ") - Ein in Steisslage herausgezogenes Kind zeigte 4 Tage nach der Geburt eine Verkürzung des rechten Kopfnickers und dabei eine Verkleinerung und Abplattung der entsprechenden Gesichtshälfte (eine ähnliche angeborene Kleinheit der betreffenden Gesichtshälfte bei angeborener Kopfnickerverkürzung beschreibt übrigens schon $\mathrm{B}$ ou vier, a. a. O., S. 88) - 7 Monate später waren Schiefhals und Asymmetrie des Kopfes von selbst verschwunden. - Schmidt nimmt nun an, dass die Difformität durch andauernde üble Haltung des Körpers in der Gebärmutter entstanden ist, wie wir dies z. B. beim Pes varus voraussetzen.

,Will und muss man nun über die Haltung des Kindes, die einen Schiefkopf veranlassen könnte, näher ins Klare zu kommen suchen, so möchte ich darauf aufmerksam machen, dass, wie bekannt, der Schiefkopf meistentheils an in Steisslage geborenen Kindern zur Beobachtung kommt, und zweitens, dass meistens der rechte Sterno-cleido-mastoideus der verkürzte ist. Man muss ferner berïcksichtigen, dass die in Steisslage, wie alle überhaupt in Verticallagen getragenen Kinder meist den Rücken nach vorn gerichtet haben (I. Lage). Stellt man sich solchermaassen das Kind auf dem Beckeneingange der Mutter sitzend, mit dem Scheitel die Spitze des Fundus uteri berührend vor, so glaube ich, dass die in der rechten Seite und der Mittelpartie der mütterlichen Bauchhöhle und oberhalb des Fundus uteri lagernde Leber der Mutter nicht ohne Einfluss auf die Haltung. des Kindskopfes bleiben können wird, das in den letzten Schwangerschaftsmonaten den reifen Körpermaassen sich nähernde Kind erreicht miț ,seinem oberen grossen Theil" die Gegend des mütterlichen Rippenbogen, und es ist eine alltägliche Klage der Hochschwangeren, dass das obere Kindsende sie empfindlich gegen und unter die Rippen, meist - bei den I. Lagen - die rechten, stösst. Bei Streckbewegungen des Kindes, oder wenn die Mutter sich bückt, wird das Gegenstossen des Kindes gegen die obere Uteruswand und deren obere Nachbartheile besonders stark werden. Ein in erster Steisslage liegendes Kind wird nun in der natürlichen Haltung seines Kopfes, wenn dieser etwa in der Gegend des mütterlichen rechten Hypochondriums steht, durch die der Ausstreckung des Kinder-

1) Meinhard Schmidt, Zum Kapitel des Schiefhalses. Centralbl. f. Chir. 1890, Nr. 30. 
nackens hindernd im Wege stehende Leber der Mutter schwer beeinträchtigt werdeñ, und zwar wird es mit seinem Kopfe, da die Unterfläche der Leber von rechts aussen unten schräg nach oben medianwärts und links verläuft, genöthigt sein, den von dieser Fläche nach der widerstandsfreien Seite abbiegenden Kopf nach der linken Seite der Mutter oder nach seiner eigenen rechten Seite der rechten Schulter zu seitlich zu neigen. Ist diese erzwungene Neigung eine sehr bedeutende und nachdrückliche, so wird die rundliche Wölbung der rechten Schulter, besonders des Humeruskopfes, sich mehr oder weniger in die aufgedrängten seitlichen Gesichtstheile am Ohre und Kiefergelenke eindrücken, so eine Gesichts- bez. Schädelasymmetrie wie in unserem Falle schon intrauterin herbeiführend. Denken wir uns dagegen das Kind in II. Steisslage liegend (Rücken nach hinten), so wird ceteris paribus dasselbe zwar wieder nach der linken Mutterseite mit dem Kopfe ausweichen, die Kindsschulter aber, der jetzt der Kopf sich näherte, wird auch die linke sein, es wird das linksseitige Caput obstipum präformirt werden, und die Richtigkeit meines Erklärungsversuches vorausgesetzt, wird dasselbe ebensoviel seltener im Vergleiche zum rechtsseitigen zu erwarten sein, als die II. Steisslage seltener als die erste ist.

Es könnten und werden auch vielleicht gegen diesen neuen Erklärungsversuch der Entstehung des Schiefkopfes Einwendungen erhoben werden, vor allem die, dass der Fundus uteri nicht unter die Leberunterfläche fixirt, vielmehr unter derselben nach vorn vorzuschlüpfen im Stande ist, oder gar der Norm gemäss schlüpfen muss, so dass der oben stehende Kindskopf wenig oder gar nicht an die Leber stossen kann. In der Regel wird dieses gewiss nicht der Fall sein, aber unter Umständen - und zwar besonders wo die Mutter sehr straffe Bauchdecken hat (ältere Erstgebärende), so dass der Uterus schlecht nach vorn schlüpfen kann, oder etwa bei spärlichem Fruchtwasser - glaube ich an die Annehmbarkeit eines gewissen Grades von solcher „Einkeilung“ sowohl des graviden Uterus als des in ihm getragenen Kindes zwischen der mütterlichen Leber und dem Becken, womit die Voraussetzung für die Möglichkeit meiner Vermuthung gegeben ist. Es sollte mich freuen, wenn letztere von den Fachgenossen für annehmbar befunden würde."

Die vorstehenden Seiten enthalten wohl Alles, was von maassgebenden Forschern über die Entstehung des sogen. angeborenen 
myogenen Schiefhalses geäussert worden ist. Auf Hypothosen sind die Anhänger der einen, wie diejenigen der anderen Ansicht angewiesen. Um auch die geringste Missdeutung auszuschliessen, wurden die Abschnitte wörtlich gegeben, welche von den die Genese betreffenden. Vermuthungen handeln.

Seit nahezu einem Jahrzehnt war nunmehr meine Aufmerksamkeit auf den Gegenstand gerichtet; die Erfahrungen, welche wir an der Bonner Klinik sammeln konnten, haben mich zu einem Anhänger der von Stromeyer, wenn auch nicht unter voller Begründung aufgestellten Lehre gemacht. - Mit Stromeyer bin ich zunächst der Ansicht, dass bereits intrauterin eine abnorm geringe Längenentwicklung des Kopfnickers statthaben kann und dass somitein auf Entwicklungsstörung beruhendes Caput obstipum musculare vorkommt. - Nicht anders kann der Fall aufgefasst werden, den Heusing er ${ }^{1}$ ) beschrieb:

„Merkwürdige angeborene Missbildung des Musculus sternocleido-mastoideus. - An einem einige Tage alten Kinde, welches mir in diesem Winter anf die anatomische Anstalt geliefert wurde, fiel mir die Richtung des Kopfes nach der linken Schulter hin auf; bei näherer Untersuchung ergab sich, dass der Musculus sterno-cleido-mastoideus die Ursache dieser Verkürzung war; denn während er auf der rechten Seite normal und $9 \mathrm{~cm}$ lang war, war er auf der linken Seite nur $6 \%_{2} \mathrm{~cm}$ lang, und bestand nicht aus Muskelsubstanz, sondern aus einer weissen, weichen Sehnensubstanz. Seine Insertionen waren normal." - Aus den Bemerkungen, welche Adelmann²) zu demselben Präparate giebt, ist ersichtlich, dass auch die Wirbelsäule verbildet war, besonders aber, dass alle übrigen Halsmuskeln der linken Seite, selbst der Musculus cucullaris, kürzer waren.

Die vorstehende Beobachtung gehört zweifellos zu den seltensten Missbildungen. Es war uns nicht möglich, noch einen weiteren Leichenbefund in der Literatur aufzufinden. In zwei anderen Fällen scheinen die Neugeborenen nur während des Lebens untersucht worden zu sein, und zwar von Bednar und G. Fischer. -

1) Berichte von der Königlichen anthropotomischen Anstalt zu Würzburg 1826 , S. 42 .

2) Bei Ammon, Die angeborenen chirurgischen Krankheiten d. Menschen, 1842. S. 59. 
Bedin a r ') schreibt: Bei einem schwächlichen Kinde hatte man nebst der Verkürzung des linken Musculus sterno-cleido-mastoideus, Lähmung der linken Gesichtshälfte, Verkümmerung des linken Ohres, Spaltung des Hinterhauptsbeines und Hydrorrhachie zu beobachten Gelegenheit gehabt. - Fischer (a. a. O., S. 34) sah bei einem in Steisslage geborenen Kinde neben Caput obstipum gleichzeitig eine Contractur des Wadenmuskels (Pferdefuss) und der Pronatoren des rechten Vorderarmes.

Wohl möchte man besonders in Hinsicht auf letzteren Fall daran denken, dass eine Raumbeschränkung in der Gebärmutter bei zu wenig Fruchtwasser, oder verhältnissmässig zu starker Entwicklung des Kindskörpers, durch Druck Anlass zur Missbildung gegeben habe, wie wir dies auf Grund kaum anfechtbarer Beweisstïcke für den congenitalen Klumpfuss annehmen. - Auffällig bliebe es dann zunächst, dass ausser der Beobachtung Fischer's keine zweite bekannt wurde, in welcher die nicht zu übersehende Coincidenz des Caput obstipum mit dem so häufigen angeborenen Klumpfusse sich gefunden hätte. Zwar sah ich einmal eine mässige, seit der Geburt bestehende rechtsseitige Kopfnickerverkürzung bei einem $11 \frac{1}{2}$ jährigen Knaben mit einem anderen angeborenen Leiden zusammen, aber mit einem solchen, bei dem wohl dio Heredität, nicht aber Beengung im Uterus eine Rolle spielt, nämlich mit einer beiderseitigen Luxatio coxae. Das Kind entstammte einer Ehe zwischen Blutsverwandten und bot ausserdem die Erscheinungen der spastischen Gilederstarre. - Noch auffalliger ist es aber dann, ja geradezu unerklärlich, wie es möglich war, dass keine Verkürzung der Kopfnicker gefunden worden ist in den von Zuckerkandl und Ribbert beschriebenen Fällen, wo infolge intrauteriner Raumbeengung eine Trichterbrust entstand indem der Unterkiefer unter starker Biegung des Kopfes gegen das Sternum angedrückt wurde. ${ }^{2}$ ) - Auf meinen Wunsch sah Ribbert das betreffende Präparat nochmals an und stellte ein durchaus normales Verhalten beider Kopfnicker fest.

Wir wissen im Uobrigen, dass im Fruchthalter während der letzten Monate der Schwangerschaft der Kopf anhaltend so vorniiber gebeugt liegt, dass unter völliger Entspannung der vorderen Halsweichtheile das Kinn mit der oberen Brustbeingegend in Be-

1) Die Krankheiten d. Neugeborenen u. Säuglinge. Wien 1853. Bd.IV, S. 50.

2) Zuckerkandl, Eine merkwürdige Difformität. Wiener med. Blätter 1880, Nr. 50. - Ribbert, Zur Aetiologie der Trichterbrust. Deutsche med. Wochenschrift 1884, Nr. 33 . 
rubrung ist. Eine anbaltende Nöherung den Ansatzpunkte der Sterno-cleido-mastoidei ist also physiologisch für diese Zeit. Will man daher von einer grob-mechanischen Erklärung nicht ganz absehen - was für unseren Fall, sowie für den von Bed nar wohl das Beste ist -., so muss, freilich ohne Rücksichtnahme auf den negativen Befund bei angeborener Trichterbrust, angenommen werden, dass durch besonders starken Druck' der Kopf seitlich gegen die Schulter angepresst war. Ausser der sonst vereinzelt dastehenden Beobachtung Fischer's könnte dann hierher das gehören, was M. Schmidt (a.a. 0., S.571) an dem 7 Tage alten Kinde sah: „Der Kopf desselben stand stark nach rechts auf die Schulter geneigt, das Kinn etwas nach links und oben gedreht. Die rechte Halsseite erschien etwas magerer und kürzer als die linke und insbesondere der Kopfnicker rechterseits knapper, kürzer, härter gespannt als der linke. Von einem Hämatom an demselben keine Spur. Ferner war am Kopfe die Gegend des oberen Theiles des Unterkieferastes, des Ohres etwa bis zum äusseren Gehörgange und die Basis der Hinterhauptsgegend fast bis zur Protuberantia occipitalis deutlich und tief grubenförmig vertieft, diese ganze Partie verkleinert und verkümmert erscheinend, als wenn sie durch den Druck eines rundlichen eiförmigen Körpers hier zur Atrophie gebracht worden wäre. Die Asymmetrie der beiden Kopfhälften war sowohl beim Anblick von vorn wie von den Seiten höchst augenfällig. Dabei war aber der Kopf mit geignetem Druck leicht ganz gerade einstellbar."

Es bleibt indess bei dieser Beobachtung gerade auffällig, dass der Kopf so leicht gerade zu richten war, hochgradig war die Kopfnickerverkürzung keinesfalls. Auch der spontane Rückgang aller Erscheinungen, besonders der Asymmetrie des Kopfes, ist nicht ganz. gewöhnlich. Ich habe einige angeborene, anscheinend intrauterin entstandene Schädelasymmetrien gesehen und dabei verfolgt, wie erst im Laufe der Jahre eine geringe Besserung sich vollzog, und möchte wenigstens darauf hingewiesen haben, dass es eigentlich annehmbarer wäre, die Veränderungen in dem Falle Sch midt durch den Druck zu erklären, welcher fast einen ganzen Tag hindurch bei der Geburt den Kopf gegen die Schulter anpresste. - Jedenfalls können wir aber mit Schmidt's oben wörtlich angeführter Anschauung vom Zusammenhange der Steisslage und der einseitigen Muskelverkürzung nicht einverstanden sein. Die letztere kommt, wenn überhaupt intrauterin, dann jedenfalls ziemlich früh und zu einer Zeit zu Stande, in welcher von einer bestimmten Lage des in einer verhältnissmässig sehr grossen Menge 
Fruchtwassers schwimmenden Embryo noch gar keine Rede ist. Die Kopfnicker entwickeln sich sehr frühzeitig, während andere Halsmuskeln viel später erscheinen (G. Fis cher). Veränderungen, wie sie Heusinger sah, entstehen keinesfalls in den letzten Monaten der Schwangerschaft durch Entspannung eines nur mehr wachsenden, nicht aber mehr in der Anlage begriffenen Muskels. - Die Entwicklungsstörungen des Falles Bed na r gohören zweifelsohne der ersten Periode an.

Wenn man schon einen Nexus zwischen Schiefhals und verkehrter Kindeslage bei der Geburt annehmen will, dann würde eher daran zu denken sein, dass die aus unbekannten Gründen während der ersten intrauterinen Entwicklung entstehende einseitige Kopfnickerkürze die Ursache einerschliesslichen abweichende Einstellung in Steisslage, mehr noch in Schulterlage, vielleicht auch in Ohrlage abgiebt. Ein solcher, von den Geburtshelfern bisher noch nicht gewürdigter Vorgang wäre wenigstens nicht ohne weiteres von der Hand zu weisen. Aber es kam weder anderen Beobachtern, noch mir kaum je eine Verkürzung vor, durch welche die Ansatzpunkte des Sternocleido-mastoideus einander näher waren, als dies bei der normalen intrauterinen Kopfhaltung während der letzten Monate der Fall ist. Eine Abweichung nach rechts oder links besteht bei schiefhalsigen Kindern auch im ersten Lebensjahre nicht für den zur Brust geneigten Kopf; sie tritt erst beim Aufrichten desselben in die Erscheinung. Im Fruchthalter wird die einseitige Muskelkürze deshalb für gewöhnlich bedeutungslos bleiben und erst dann zur Geltung kommen, wenn für irgend eine Einstellung vor der Geburt, oder für eine solche nach Beginn der Wehenthätigkeit, zumal nach dem Fruchtwasserabflusse, eine Dehnung der betreffenden Halshälfte erforderlich würde. Dann mag schon das Untere nach oben kommen und der Steiss erscheinen, oder es mag die Schulter sich einstellen und durch Wendung eine Beckenendlage hergestellt werden müssen. - Nun ist aber ganz besonders zu betonen, dass nur in der Minderzahl der Fälle bei ursprünglicher oder kiinstlich geschaffener Einstellung des unteren Kindsendes die Ausziehung erforderlich ist; für gewöhnlich verläuft die Ausstossung von selbst ohne Zug am Rumpfe, obne gewaltsame Entfernung des Kopfes rom Rumpfe. Wo bleibt dann das Gros der intrauterin entstandenen Schiefhälse, bei denen nicht gezogen wurde, bei denen jene doch immerhin mehr zufällige Bceinflussung der Einstellung nicht stattand? Man 
wird auf den Fall Heusinger hinweisen, er ist aber auch bisher der einzige, bei dem zweifellos zur Zeit der Geburt der Kopfnicker verkürzt war und alte Gewebsveränderungen zeigte. Klinisch wurde wohl des Oefteren festgestellt, dass die Eltern gleich nach der Geburt eine Abweichung einer Halsseite entdeckten, dagegen wurde bisher noch nie an dem eben geborenen Kinde die Muskelverkürzung als Ursache einer Torticollis von einem Arzte festgestellt. Geradezu auffällig ist eine diesbezügliche Angabe von Guyon 1): „Le relevé de Chaussier à la Maternité, dans le but de rechercher la proportion des monstruosités aux naissances, porte sur cinq années comprenant 23293 enfants. Sur ce nombre 132 ont présenté des monstruosités diverses et dans quelques cas multiples; a ucun n'était affecté de torticolis. Il manque donc pour établir le fait la seule preure positive une observation prise à la naissance. Si l'on réfléchit maintenant aux obstacles bien connus, qu'apportent à l'accouchement les positions inclinées du sommet et que dans le cas particulier cette inclinaison devrait être irréductible, ou devra être étonné de n'avoir jamais entendu signaler cette cause parmi celles si nombreuses de la dystocie, et l'on sera bien près de conclure que si le torticolis congénital existe, il ne se montre jamais qu'à un bien faible degré. Mais faudrait-il encore le constater au moment de l'accouchement..: Die Zahl der untersuchten Fälle wird ein Jeder für genügend gross erachten.

Fügen wir hier gleich noch hinzu, dass C. Ruge, welcher lange Jahre hindurch die Sectionen Neugeborener an der Königlichen Entbindungsanstalt zu Berlin machte, nie eine angeborene Verkürzung des Kopfnickers fand.

Nach dem Vorstehenden erkennen wir, dass es jedenfalls kaum angehen wird, auch nur für einen grösseren Procentsatz der angeborenen Schiefhälse intrauterin entstandene Kopfnickerkürze als Ursache anzunehmen. Sonst kommt eben eine mehr oder minder gewagte Hypothese zur Stütze der anderen, und wir müssen schliesslich noch voraussetzen, dass mit oder ohne besonderen Zug die intrauterin verkürzten Kopfnicker der Regel nach bei der Geburt zerreissen und nur ganz ausnahmsweise unverletzt bleiben (Fall Heusinger, Schmidt). Ehe wir uns entschliessen

1) Torticollis in Dict. encycl. des sciences médicales, III série XVII p. 671.1887. 
können, so weit mit auf das Gebiet der Vermuthungen zu gehen, möchten wir die Zerreissung des vorher verkürzt gewesenen Muskels als bei der Geburt entstanden auch nur in einem Falle erwiesen sehen. Bis dahin geben wir zu, dass vereinzelte Fälle ron wirklich intrauterin entstandenen Torticollis vorkommen, betonen jedoch, dass eine Erklärung für das Entstehen der Kopfnickerverlkürzung bisher noch vollständig fehlt.

Die Autoren, welche für die intrauterine Genese der Verkürzung des Kopfnickers sind, haben auch noch einen Punkt von Wichtigkeit gänzlich ausser Acht gelassen; derselbe betrifft die Veränderungen an den übrigen Weichtheilen des Halses und am Skelett. Beim Pes varus congenitus ist die Verkürzung der Muskeln an der Hinterseite des Unterschenkels den Veränderungen am Fussgerüste gegenüber eher Neben- als Hauptsache. Nun finden wir wirklich in dem von Heusinger untersuchten Falle Verkürzung sämmtlicher Muskeln der betreffenden Seite und eine Abweichung der Halswirbelsäule erwähnt. Es ist nicht recht einzusehen, aus welchen besonderen Gründen sonst der Kopfnicker allein schrumpfen soll; und gesetzt auch dies, so scheint es kaum denkbar, dass die abnorme Kürze des Muskels ohne Einfluss auf die Bildung der Halswirbel und der Kopfknochen bleiben kann. Ein jeder Chirurg kernt die secundären Veränderungen, welche in Form einer typischen Skoliose durch einseitige dauernde Verkürzung des Sterno-cleido-mastoideus am Skelett, und zwar besonders am Kopfe, veranlasst werden. Man kann sie von ihren ersten Anfängen an im extrauterinen Leben verfolgen; der Mechanismus ihrer Entstehung ist von mir (a. a. O.) dargethan worden. Die von Schmidt beschriebene Ungleichheit, noch mehr die einseitige Missbildung am Kopfe des von Bednar untersuchten Kindes hat nichts mit jener typischen Skoliose gemein, welche nach allen Gesetzen des Wachsthums erfolgen muss, und zwar in höchstem Grade, wenn während der intrauterinen Entwicklung längere Zeit hindurch der Kopf anhaltend einer. Schulter stark genähert ist. - In dem Falle Heusinger erfahren wir nichts über das Verhalten des Kopfes. Für jede künftige Beobachtung von wirklich angeborenem Schiefhalse verdienen diese Verhältnisse genaue Würdigung. -

Wenden wir uns nunmehr zu der durch Stromeyer begründeten Anschauung, welche das angeborene myogene Caput obstipum auf eine Verletzung bei der Geburt zurückführt. Klar und bündig 
wurde dieselbe dargethan durch Volkmann '); die Worte dieses Meisters der Darstellung mögen hier zurechtweisen: ,, Dass die Halsmuskeln bei schwierigen, zumal operativen Geburten sehr häufig stark gezerrt werden müssen, ist selbstverständlich. Indess handelt es sich in diesen Fällen wohl häufiger um partielle Einrisse und fasciculäre Rupturen, als um vollständige Continuitätstrennungen, wenngleich auch die letzteren öfter vorkommen mögen. Wird die Störung nicht übersehen, was freilich das Häufigere ist, so dass erst später die Folgen hervortreten, so bemerlit man bald nach der Geburt eine spindelförmige Geschwalst am Muskel, die zum Theil aus ergossenem Blute, zum Theil aber auch aus dem entzündlich geschwollenen Muskel selbst besteht und sich innerhalb der nächsten Zeit in einen festen, knorpelartigen Knoten umwandelt. Während dieser langsam resorbirt wird, entsteht nun in einzelnen Fällen die Contractur, die ihren Grund theils in der cicatriciellen Schrumpfung, theils in der von den ersten Lebenstagen an instinctiv eingenommenen, den Muskel erschlaffenden, schiefen Stellung des Kopfes findet."

St rom ey er hatte die Zerreissung meist dicht über der Sternalinsertion gefunden; Volkmann fühlte den Knoten in wenigen Fällen in den mittleren Partien des Muskelbauches, erwähnt aber auch Anschwellungen des Muskels in seiner ganzen Ausdehnung und Bildung eines gleichmässigen knorpelartigen Stranges, einen Zustand, der von französischen Forschern als Sklerose des M. sterno-cleido-mastoideus bezeichnet wird.

Die zuerst auftretende Schwellung bezeichnet man gewöhnlich als Hämatom des Kopfnickers nach Bohn, welcher über ,das Hämatom der Sterno-cleido-mastoidei bei Neugeborenen" zwei Mittheilungen machte (Deutsche Klinik 1864, XVI, S. 267 ff. und 507). In der ersten findet sich das literarische Material, welches nebst eigenen Beobachtungen der Schilderung Volkmann's zu Grunde liegt. Bohn konnte freilich keinen bestimmten Fall beibringen, in welchem das Muskelhämatom von einem Arzte gleich nach der Geburt untersucht wurde.

Auch bei Chelius ${ }^{2}$ ) bleibt es zweifelhaft, ob er nach eigener Anschauung berichtet: „Nach schwierigen Geburten und nach Anwendung der Zange bemerkt man manchmal eine kleine, runde,

1) Pitha-Billroth's Handbuch der Chirurgie: Die Kraukheiten der Bewegungsorgane. S. 872 . merkung.

2) Handbuch der Chirurgie I. 7. Auf. Kapitel: Capnt obstipum. An- 
bläuliche, teigige Geschwulst oberhalb des Schlïsselbeines, dem Verlaufe des Kopfnickers entsprechend, die nach ihrem Verschwinden eine harte, dicke Substanz fühlen lässt und durch partielle oder totale Zerreissung des Muskels bedingt ist."

Die erste Schilderung des anatomischen Befundes wurde von Skrzeczka ') gegeben von drei Kindesleichen, wolche zur gerichtlichen Autopsie kamen. Zwei der Kinder schienen in Schädellage geboren, das eine davon wies ein Extravasat in der Muskelscheide beider Kopfnicker auf, beim andern fand sich der Bluterguss am rechten Kopfnicker. Skrzeczka glaubt, dass die Blutaustritte bei angewandter Selbsthiülfe der Gebärenden durch Dehnung bezw. Streckung des Halses zu Stande gekommen waren. Im dritten Falle fand sich ein Hämatom des rechten Sterno-cleido-mastoideus; es hatte sich um Beendigung einer Fusslage durch eine dritte Person gehandelt.

Drei weitere Leichenbefunde, in denen sich bei Neugeborenen Blutungen im Kopfnicker fanden, gab dann Fasbender ${ }^{2}$ ) in seinem Vortrage: Verletzungen des Kopfnickers bei der Geburt. Der Vortrag knüpft übrigens an einen Fall an, der auch ron Petersen in nähere Erwägung gezogen wurde. Zwölf Stunden nach einer bei Beckenenge durch Wendung und Ausziehung beendigten Geburt fand sich eine bläuliche teigige, bei Berührung empfindliche Geschwulst, welche die untere Hälfte des rechten Kopfnickers einnahm. Die Schwellung ging bis auf eine kleine Härte zurück. Man wird nichts Besonderes darin finden können, wenn ganz im Anfange der Kopf nach der gesunden Seite hinübergedrängt war. Aehnliches giebt $\mathrm{B}$ ohn an, als durch einen grossen Bluterguss in die Muskelscheide bedingt; auch Dieffenbach sah infolge einer primären Entzündungsgeschwulst eine Neigung nach der gesunden Seite. Der Kopf des Kindes, über welches Fasbender berichtete, blieb nach Angabe der Mutter bis zu dem drei Monate nach der Geburt erfolgten Tode nach der linken Seite hin verzogen. - Ich habe Anlass genommen, Eltern über ihre Auffassung der Stellungsveränderung beim Caput obstipum zu fragen und ebenso häufig bei rechtsseitigen Torticollis z. B. die Antwort bekommen, der Kopf sei nach rechts verzogen, wobei die Stellung des Ohres Berücksichtigung fand, als auch, dass er nach

1) Extravasate an den Kopfnickern bei Neugeborenen infolge von Selbsthülfe bei der Geburt. Vierteljahrsschr. f. ger. Med., N. F., X. 1.

9) Beiträge zur Geburtshülfe und Gynäkologie II, 3. Heft, S. 170. 1873. 
links verzogen sei, dann wurde besonders auf die Lage des Kinnes geachtet. Dieselben verschiedenen Angaben erhält man auch, wenn man einem Laien gegenüber den Kopf in die Stellung des Caput obstipum bringt.

Von grösster Bedeutung für unsere Fragen ist eine Arbeit,

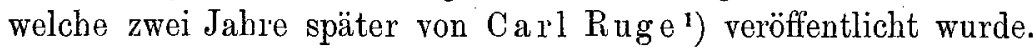
Wir heben zunächst einen Satz hervor: „Angeborene Verkürzungen des Kopfnickers (Dieffenbach, Stromeyer) habe ich bis jetzt noch nicht nachweisen können, und muss man fürs erste Veränderungen dieses Muskels hauptsächlich als acquirirt, aus den angeführten Läsionen entstanden, annehmen." - Die betreffenden Läsionen, welche $R$ uge unter etwa 64 Fällen von Extractionen $18 \mathrm{Mal}$ beobachtete, bestanden in Blutung in den Kopfnickern, über denselben und im umliegenden Bindegewebe. Es handelte sich im Muskel, und zwar an den verschiedensten Stellen um kleine, mikroskopisch auch leicht nachweisbare Zerreissungen, die sich zuweilen auf einzelne Muskelfasern allein beschränkten, oder un solche, welche grössere Partien einnahmen, grössere Blutaustretungen im Muskel bedingten; selten fanden sich völlige Durchreissungen. Die betreffenden Muskelpartien waren bald mehr, bald weniger geschwollen und durch rothbläuliche Färbung gekennzeichnet, ,besonders im Gegensatze zu den normalen, dagegen blass erscheinenden intacten Stellen". Die äusserlichen Zeichen, besonders die Anschwellung, waren dabei nicht auffallend, auch eine bläuliche oder blaugraue Färbung der Haut trat nur hervor, wenn neben den Muskelverletzungen im Unterhautgewebe Blutergüsse lagen. - Wir sehen also, dass R u ge weder den Muskel congenital zu kurz gerissen fand, noch am zerrissenen Kopfnicker Zeichen sah, welche als die einer intrauterinen Verkürzung zu deuten waren. Seine Schilderung von der Geringfügigkeit äusserlich wahrnehmbarer Veränderungen macht es auch begreiflich, warum nur in seltenen Fällen das eigentliche Kopfnickerhämatom klinisch entdeckt wird. - Es ist nun weder von Stromeyer, noch von irgend einem wissenschaftlichen Anhänger seiner Theorie jemals behauptet worden, dass ein jeder Kopfnickerriss eine bleibende Verkürzung zur Folge haben müsse.

1) Ueber die Verletzungen des Kindes durch die Extraction bei ursprünglicher oder durch Wendung herbeigeführter Beckenendlage, nebst kurzer Beleuchtung der Extractionsmethoden. Zeitschrift f. Geburtshülfe u. Franenkrankheiten 1875, Bd. I, S. 68. 
Das Gegentheil hatten schon Dieffenbach und Bohn gezeigt, und selbst Stromeyer berichtet über einen Fall, in dem sich die callöse Geschwulst zertheilte. Die Bedingungen, unter denen die Umwandlung des verletzten Muskels zu einem unten gegabelten derben runden Strange erfolgt, sind bis jetzt unbekannt; vielleicht gewinnen wir im Laufe der Zeit eine bessere Einsicht derselben, wenn die eine Autopsia in vivo gestattende offene Durchschneidung Volkma nn's') häufiger geübt wird. - In ungefähr 12 Fällen, bei denen der verkürzte Muskel behufs Durchtrennung in offener Wunde von Volkmann blossgelegt wurde, bot sich ein sehr verschiedener Befund. Bald fand sich weder eine Narbe, noch eine einer Inscriptio tendinea ähnliche Unterbrechung, die als Folge eines früher erlittenen Trauma aufgefasst werden konnten. Ein ander Mal boten sich die schwersten Veränderungen, wie sie nach besonders heftigen und ausgebreiteten entzündlichen Vorgängen zurückbleiben. ,In der Ausdehnung mehrerer Zolle zeigt der Muskel eventuell gar keine contractile Substanz mehr, sondern ist in eine weisse, gefässarme, sehnig-schwielige Masse verwandelt. Die Muskelscheide ist nicht mehr darstellbar, der verlängerte Muskel mit narbigen Strängen, die theils zur Seite strahlen, theils in die Tiefe führen; verwachsen. Die Gefässscheide verdickt, schwielig. Unter dem Mikroskop fand sich in zwei Fällen in der mitten aus dem Sterno-cleido-mastoidu herausgenommenen schwieligen Masse auch nicht eine einzige Muskelfaser mehr." Dazwischen kamen als Uebergänge Muskeln mit Sehnenflecken oder einzelnen schwieligen Strängen vor.

Volkmann hebt nun hervor, dass die geringfügige Verletzung, welche sich als Kopfnickerhämatom darstellt, selten ein Caput obstipum zur Folge haben wird, obwohl er auch dies ein paar Mal gesehen habe; in der Regel liegen der bleibenden Muskelverkürzung subcutane Zerreissungen von Muskeln und Fascien $\mathrm{zu}$ Grunde, gegenüber welchen das gewöhnliche Hämatom dos Sterno-cleido-mastoideus nur eine leichte Störung ist. Die schwereren Veränderungen sind nur durch active, und zwar sehr lebhafte Vorgänge zu erklären: ,du rch fehlerhafte Haltung, Raumangel in utero u. s. w. kann Derartiges nie zu Stande kommen."

1) Das sogenannte angeborene Caput obstipum und die offene Durchschneidung des M. sterno-cleido-mastoideus. Centralbl. f. Chir. 1885, Nr. 14. 
Die Angaben Volkmann's wurden vor Kurzem wieder bestätigt in der Mittheilung seines Schülers Vollert ${ }^{1}$ ); dieselbe bringt auch zwei Photogramme von Querschnitten, in denen man eine Wucherung des Bindegewebes erkennt, welche auf Kosten der nur noch spärlich vorhandenen atrophischen Muskelsubstanz stattgefunden hat.

In den beiden letzterwähnten Arbeiten ist das Alter der Kinder nicht erwähnt, bei deren Operation die Beobachtungen gemacht, bezw. die Präparate gewonnen wurden. An unserer Rlinik wurde bisher im Ganzen selten nach Volkmänn operirt. Der letzte Fall bot Gelegenheit zur Exstirpation eines Stückes etwas unterhalb der schon äusserlich fühlbaren Anschwellung im verkürzten Kopfnicker bei einem 6 Monate alten weiblichen Kinde. Dasselbe war wegen Querlage gewendet und dann unter grossen Schwierigkeiten ausgezogen worden. Schon am 10. Tage war die schiefe Haltung des Kopfes aufgefallen und eine abnorme Verdickung am Halse rechts bemerkt worden. Hier war der Kopfnicker bei der Aufnahme des Kindes in einen derben runden Strang verwandelt, der etwas oberhalb seiner Mitte eine kleine Verdickung erkenuen liess und nur halb so lang als der Muskel der anderen Seite war. Bei der Operation fanden wir beweglich in eine Scheide lockeren Bindegewebes eingebettet einen sehnig umgewandelten Strang, welcher nach Exstirpation eines kleinen Stückes quer durchtrennt wurde. - Prof. Köster, welcher der schwebenden Frage lebhaftes Interesse entgegenbringt, untersuchte das Präparat und gab folgenden Bescheid:

„Die Frage, ob Degeneration des Muskels oder ob traumatische Zerstörung mit Vernarbung, entscheidet sich bei vorliegendem $\mathrm{Ob}$ ject unzweifelhaft in letzterem Sinne.

Das bald sehr feingekräuselte, bald derbfaserige sklerotische Bindegewebe verläuft in dem Object in bald breiteren, bald schmäleren Zügen nach allen Richtungen, woraus hervorgeht, dass es von verschiedenen Punkten ausgesprosst ist. In derselben Weise verhalten sich die nicht spärlichen Gefässe.

Entscheidend ist jedoch erst das Vorhandensein von einzelnen Bruchstücken von Muskelfasern, die wie zerstreute Splitter, aber nur in den äusseren Partien, jedoch allseitig von Bindegewebe

1) Zur Operation und pathologischen Anatomie des congenitalen Caput obstipum. Centralbl. f. Chir. 1890, S. 38. 
umschlossen, kreuz und quer in demselben liegen. Die einzelnen Muskelfasern zeigen keinerlei Art der Degeneration, nur ist die Querstreifung vielfach undeutlich. Einzelne haben mehr Kerne als normal, andere nicht. Viele spitzen sich an dem einen Finde $\mathrm{zu}$, sind aber dann am anderen breit und wie quer abgebrochen (nicht abgeschnitten)."

Ueber den Befund, welchen der verkürzte Muskel in späterer Zeit bietet, finden sich bisher nur zwei Veröffentlichurgen. Die eine wurde von Bouvier') gegeben. Bouvier untersuchte die Leiche eines 22 jährigen Mädchens mit angeborenem Schief halse und fand den rechten Kopfnicker viel dünner und schmäler als den linken, auch fast nur halb so kurz; die Portio clavicularis maass rechts 3 Zoll Länge und links 5 Zoll; die Portio sternalis, rechts 4 Zoll 3 Linien lang, mass links 7 Zoll. Auf der gesunden Seite boten die Muskelfasern den gewöhnlichen Anblick, rechts waren sie blass und in so geringer Menge vorhanden, dass die Hauptmasse des Muskels aus sehnigem Gewebe bestand.

Ausserdem wurde von mir die Leiche einer 44 Jahre alten Frau mit congenitalem muskulären Schiefhalse ${ }^{2}$ ) in Rostock untersucht: „Der rechte Kopfnicker maass an seinem lateralen Rande 16, an seinem inneren Rande $18 \mathrm{~cm}$, dicht oberhalb der Gabelung war derselbe $2,5 \mathrm{~cm}$ breit; die Portio sternalis war im Verhältniss zur Clavicularportion sehr schmal. Im Ganzen war der Muskel glatt und zeigte keine Structurveränderungen. Der linke Kopfnicker war in einen fast durchweg sehnigen Strang verwandelt; in seinem oberen, ungetheilten Abschnitte sowohl, als auch in den beiden unteren Portionen drehrund; er maass am lateralen Rande $8,5 \mathrm{~cm}$, am medialen $10,5 \mathrm{~cm}$ Länge und war dicht oberhalb der Theilung $1,2 \mathrm{~cm}$ breit. SternaI- und Clavicularportion zeigten gleich starke Entwickelung, $1 \mathrm{~cm}$ oberhalb und unterhalb der Theilung, sowie am unteren Ende der Sternalportion fanden sich grauröthliche Längsstreifungen; hier wies das Mikroskop stark cadaverös veränderte Reste von Muskelfasern nach.“"

Wer das vorstehende Gesammtergebniss der pathologischanatomischen Forschungen über den muskulären Schiefhals vorurtheilsfrei ansieht, der wird darin nichts finden, was gegen die

1) L'expérience 1830 , T. I, p. 510.

2) 0 . Witzel, Beiträge zur Kenntniss der secundären Veränderungen beim muskulären Schiefhalse. Deutsche Zeitschr. f. Chir., Bd. XVIII. 
Annahme Stromeyer's angeführt werden kann; uns scheint im Gegentheile die Erklärung des bisher Gefundenen durch eine bei der Geburt stattgehabte Gewalteinwirkung die naheliegende, natürliche zu sein. - Nehmen wir noch hinzu, was $\mathrm{klin}$ is ch beobachtet wurde. Volkmann sah die Verkürzung im Anschlusse an das Hämatom und mehr ist überhaupt nicht zu verlangen; Chelius und Andere beschrieben die Umwandlung der Blutgeschwulst zum Bindegewebscallus; dass aber der letztere nicht nur als das zuerst Auffallende an dem schrumpfenden Muskel hervortrat, sondern auch bei ausgebildeter Verkürzung noch vorhanden war, ist eine sehr häufige Beobachtung. Die einzelnen Stadien, welche anatomisch festgestellt wurden, kehren beim Verfolgen der klinischen Befunde wieder; sie heissen: Bluterguss im Muskel, Bildung einer festen, mehr oder weniger geschwulstartigen Narbe, dann fibröse Umwandlung des Muskelrestes. Es liegt auf der Hand, dass man unmöglich den anatomischen Nachweis der drei Stadien an demselben Individuum liefern kann. Für die Beweisführung durch Beobachtungen am Lebenden muss es hier, wie auch sonst, genügen, die Uebergänge von den ersten zu den am Schlusse sich bietenden Erscheinungen an verschieden liegenden Reihen von Einzelbeobachtungen zu erkennen. Finden wir für das Ergebniss unseres Suchens Einklang zwischen dem, was die klinische Beobachtung sagt, und dem, was die anatomischen Veränderungen lehren, schon dann stehen die weiteren Schlussfolgerungen nicht mehr auf unsicherem hypothetischen Grunde. Selbst durch noch ganz andere Entgegnungen, als die bisherigen, würden sie nicht erschüttert werden können; sie brauchten nicht mehr die Stütze einer Statistik, wie wir sie zu geben in der Lage sind, auf Grund einer über neun Jahre sich erstreckenden Verfolgung der in Rede stehenden Fragen. Heredität, Gravidität, Partus fanden bei den angestellten Nachforschungen stets Berücksichtigung. -

Es ist mehrfach beobachtet worden, dass mehrere Geschwister an congenitalem Schiefhalse litten; so erwähnt Petersen, dass zwei Kinder derselben Frau das Leiden zeigten; G. Fischer berichtet, dass eine Frau Mutter von 7 Schiefhälsen wurde, die Geburten waren immer schwer, mehrfach war die Zange erforderlich; auch Stromeyer schon sah die Affection bei Schwester und Bruder (a. a. O., XLVII). Man hat deshalb das Moment der Heredität ins Spiel bringen wollen. Wenn man die für das Kind bestehende Nothwendigkeit, ein enges Becken passiren zu müssen, Archiv f. Gynäkologie. Bd. XLI. Hft. 1 n. 2. 
erbliche Belastung nennen will, dann waren die betreffenden Schiefhälse hereditär. Sonst bezeichnet man als hereditär Eigenthümlichkeiten, die sich von Generation zu Generation continuirlich oder discontinuirlich fortpflanzen. Was die Verhältnisse der Gravidität angeht, so wurde, wie in Fällen von Klumpfussbildung, danach gefragt, ob die Bewegungen des Kindes ungewöhnlich schmerzhaft gewesen waren oder nicht. Man nimmt ja an, dass bei einer durch Fruchtwassermangel bedingten Raumbeschränkung die Kindsbewegungen peinlicher empfunden werden. Nur in einem Falle aus der Privatpraxis Trendelenburg's wurde von der Mutter bestimmt angegeben, dass die Bewegungen schmerzhafter als sonst gewesen seien und dass nur wenig Fruchtwasser abgeflossen wäre.

Bei einem Leiden, welches von jeher von Arzt und Laien bis auf die Geburt zurückgeführt wurde, müssen im Uebrigen, wenn es nach unserer Darstellung nur ausnahmsweise wirklich intrauterin entstehen dürfte, die Vorgänge bei der Geburt von grösster Bedeutung sein. Unsere Beobachtungen, die an der Bonner chirurgischen Klinik und auf der unter Geheimrath Trendelenburg's Leitung stehenden chirurgischen Abtheilung des Johannishospitals zu Bonn gemacht wurden, umfassen im Ganzen 43 Fälle und sind in der folgenden Uebersicht kurz wiedergegeben. ${ }^{1}$ )

\section{A) Geburt in Beckenendlage beendet.}

I. Mit Kunsthülfe.

\section{a) Ausziehung nach Wendung.}

1) Knabe, 9 Jahre alt, Caput obstipum musculare sinistrum mit starker Skoliose des Gesichts - und Hirnschädels. Die Wendung wurde wegen Querlage ausgeführt. In den ersten Tagen am Halse nichts Abweichendes bemerkt; die schiefe Haltung des Kopfes bildete sich vom Ende des ersten Lebensjahres aus.

2) Knäbe, 10 Jahre alt, mit Verkürzung des linken Kopfnickers und ausgeprägter Skoliose des Schädels. Die Geburt wurde durch Wendung und Ausziehung beendet. Schiefstellung des Kopfes im vierten Lebensjahre bemerkt.

3) 9 jähriger Knabe, mit rechtsseitigem muskulären Schief-

1) Das Nähere findet sich in den Dissertationen von Fabry (1885) und Colombara (1891). 
halse und starker Schädelskoliose, wurde nach einer durch Querlage angezeigten Wendung an den Füssen ausgezogen.

4) $5^{3} / 4$ Jahre alter Knabe mit Caput obstipum musculare dextrum, seit der Geburt bestehend, die wegen Placenta praevia mit Wendung auf die Füsse ausgeführt wurde.

5) 8 Jahre altes Mädchen mit linksseitigem muskulären Schiefhalse und Skoliose des Schädels, Halses und Rumpfes; nach Wendung an den Füssen ausgezogen. Schiefe Stellung des Kopfes zuerst im Alter von 2 Jahren bemerkt.

6) Mädchen im Alter von 6 Monaten wegen ausgeprägter rechtsseitiger Kopfnickerverkürzung zur Klinik gebracht. Das Kind war wegen Querlage gewendet und unter grossen Schwierigkeiten an den Füssen ausgezogen worden. Schwere Asphyxie. Schon am 10. Tage wurde die schiefe Haltung des Kopfes und eine abnorme Verdickung am Halse rechts bemerkt. Die Asymmetrie des Gesichtes fiel den Eltern in letzter Zeit auf. Der rechte Kopfnicker ist gerade halb so lang als der linke, er bildet einen unten festen, oben etwas weicheren Strang. In seiner unteren Hälfte bei der Operation blossgelegt, erscheint der Sterno-cleido-mastoideus fast rein sehnig, jedoch nicht mit der Umgebung verwachsen. (Präparat von $\mathrm{K} \ddot{\text { sste }} \mathrm{r}$ untersucht.)

\section{b) Ausziehung bei Steiss- und Fusslage.}

1) Mädchen von 5 Jahren als zweites Zwillingskind in Steisslage herausgezogen. Kopf seit der Geburt nach links gehalten.

2) 3 Jahre altes Mädchen mit rechtsseitigem Schiefhalse wurde ohne besondere Schwierigkeit in Steisslage ausgezogen. Im ersten Lebensjahre wurde nichts Abnormes bemerkt; seit 8 Monaten tritt die schiefe Kopfhaltung hervor.

3) 8 jähriger Knabe mit sehr starker Verkürzung des rechten Kopfnickers wurde als zehntes Kind in Steisslage ausgezogen. Im Alter von 2-3 Jahren wurde die Schiefhaltung des Kopfes bemerkt.

4) Mädchen im Alter von 6 Jahren, als sechstes Kind in Fusslage geboren; der nachfolgende Kopf wurde mit der Zange entwickelt. Kurze Zeit nach der Geburt wurde rechts am Halse eine Anschwellung bemerkt; der Schiefstand des Kopfes bildet sich in dèn letzten Jahren aus. Skoliose an Kopf, Hals und Brust.

5) 7 Monate altes Kind mit Verkürzung des rechten Kopfnickers um ein Drittel; es wurde an den Füssen herausgezogen 
und zeigte von Anfang an eine schiefe Kopfhaltung; im 6. Monate wurde die Muskelverkürzung entdeckt.

6) $3^{1} / 2$ jähriger Knabe an den Füssen ausgezogen, zeigte gleich nach der Geburt eine Abnormität der rechten Halsseite. Typische Veränderungen des Caput obstipum musculare dextrum.

7) Knabe bei der Aufnahme $6 \frac{1}{2}$ Jahre alt, mit linksseitigem Schiefhalse, wurde in Steisslage extrahirt, wobei auch eine Fractur des linken Oberarmes zu Stande kam. Schädelskoliose gering.

8) 12 Jahre alter Knabe mit rechtsseitigem Torticollis und leichten skoliotischen Veränderungen am Kopfe; Steissgeburt durch Auszichung beendet.

\section{Ohne Kunsthülfe.}

1) Linksseitige Verkürzung des Kopfnickers seit der in Steisslage erfolgten Geburt bemerkt. Kind bei der Aufnahme 31/2 Jahre alt.

2) 12 Jahre alter Knabe in Steisslage geboren. Verkürzung des linken Kopfnickers. Bald nach der Geburt entwickelt sich die schiefe Haltung des Kopfes; es wurde ein Knötchen links am Halse entdeckt.

3) Caput obstipum sinistrum bei einem 10 Jahre alten, in Steisslage geborenen Knaben. Die schiefe Kopfhaltung wurde erst bemerkt, als das Kind anfing zu gehen; sie trat in den letzten Jahren stärker hervor.

4) 9 Jahre alter Knabe, in Steisslage natürlich geboren. Die ersten Veränderungen am Halse wurden im Alter von $5-6$ Monaten bemerkt, als das Kind anfing aufrecht zu sitzen; gegen Anfang des vierten Lebensjahres war der Schiefstand des Halses sehr deutlich.

5) Muskulärer Schiefhals bei einem 21/2 Jahre alten Mädchen, welches in Steisslage geboren war.

\section{B) Geburt in Schädellage beendet.}

\section{Mit Kunsthülfe.}

a) Zangenoperationen.

1) 10 Jahre alter Knabe mit hochgradigem linksseitigen Schiefhalse. Kopf mittels der Zange entwickelt, dabei auch Verletzung oberhalb des rechten Auges. Schon vom zweiten Monate an bemerkte die Mutter, dass der Kopf des Kindes zur linken Seite sich neige. 
2) 8 Monate altes männliches Kind mit Verkürzung des linken Kopfnickers. Ausziehung mittels der Zange. Seit einem Monate fällt die schiefe Kopfhaltung auf.

3) 7 Jahre alter Knabe mit hochgradiger. Verkürzung des linken Kopfnickers und entsprechenden Veränderungen am Schädel. Geburt mit Zangenanwendung. Difformität erst seit einem halben Jahre bemerkt.

4) Mädchen von 7 Jahren. Bald nach der mit Anwendung des Forceps beendeten Geburt wurde eine kleine Einbuchtung rechts am Kopfnicker bemerkt. Caput obstipum dextrum mit consecutiver Skoliose des Schädels und der Wirbelsäule.

5) Knabe 5 Jahre alt. Caput obstipum dextrum. Ausziehung mittels der Zange. Verkürzung des Kopfnickers, zuerst von den Eltern bemerkt, als das Kind 2 Jahre alt war.

6) Blödsinniger Knabe von 4 Jahren. Ausziehung mittels der Zange. Der rechte Kopfnicker ist in eine spindelförmige, sehr harte Geschwulst verwandelt, welche exstirpirt wird.

7) 3 Jahre alter Knabe mit Caput obstipum musculare dextrum wurde mittels der Zange ausgezogen.

8) 5jähriges Mädchen. Zangenoperation. Bedeutende Verkürzung des rechten Kopfnickers und hochgradige Asymmetrie des Gesichtes.

\section{b) Ausziehnngen mittels der Hand.}

1) Männliches Kind, $1 \mathrm{Jahr}$ alt, wurde von der Hebamme mit starkem Zuge entwickelt. Einige Tage nach der Geburt Anschwellung links am Halse bemerkt. 4 Wochen nach der Geburt fanden wir den linken Kopfnicker im Ganzen geschwollen und hart; oberhalb seiner Mitte bestand noch eine besondere Verdickung. Gegen Ende des ersten Lebensjahres war noch eine deutliche Verkürzung und Verdickung des Muskels vorhanden.

2) 11 Jahre alter Knabe wurde wegen Nabelschnurumschlingung um den Hals schnell ausgezogen. Die linksseitige Kopfnickerverkürzung trat zuerst hervor bei Beginn des Schulbesuches; sie hatte starke secundäre Veränderungen am Skelett des Kopfes, Halses und Rumpfes zur Folge.

3) 5 Monate altes Mädchen. Geburt ebenfalls wegen Nabelschnurumschlingung schnell beendet durch Zug am Kopfe. Vom 
15. Tage an bestand eine Neigung des Kopfes nach links hin. Wir stellten eine Verkürzung des gespannten und in seiner Mitte verdickten linken Kopfnickers fest.

\section{Ohne Kunsthülfe.}

1) Bei einem 4 Wochen alten männlichen Kinde fand sich der linke Kopfnicker verkürzt; in seinem oberen Theile, ungefähr in der Mitte anfangend, bestand eine zwei Finger breite Verhärtung. Die Geburt war schwer gewesen und in Kopflage erfolgt.

2) 3 Jahre altes Mädchen mit typischem Caput obstipum musculare sinistrum, welches seit $1 / 2$ Jahre bemerkt wurde; die Geburt ist in Schädellage ohne Kunsthülfe erfolgt.

\section{C) Geburt mit Kunsthülfe beendet, Näheres unbekannt.}

8 Jahre alter Knabe mit rechtsseitigem Caput obstipum unusculare wurde mit Kunsthülfe geboren. Nach einigen Wochen wurde eine Schwellung am rechten Sterno - cleido-mastoideus bemerkt; derselbe trat unter Zunahme der schiefen Kopfhaltung strangartig hervor. Typische secundäre Veränderungen.

\section{D) Geburtshergang unbekannt.}

In 9 Fällen war es nicht möglich, Auskunft über den Hergang der Geburt zu erhalten. Der Schiefhals bestand in 5 derselben nach rechts hin, in 3 nach links; in 1 Falle fehlt die Abgabe iber die Seite der Abweichung.

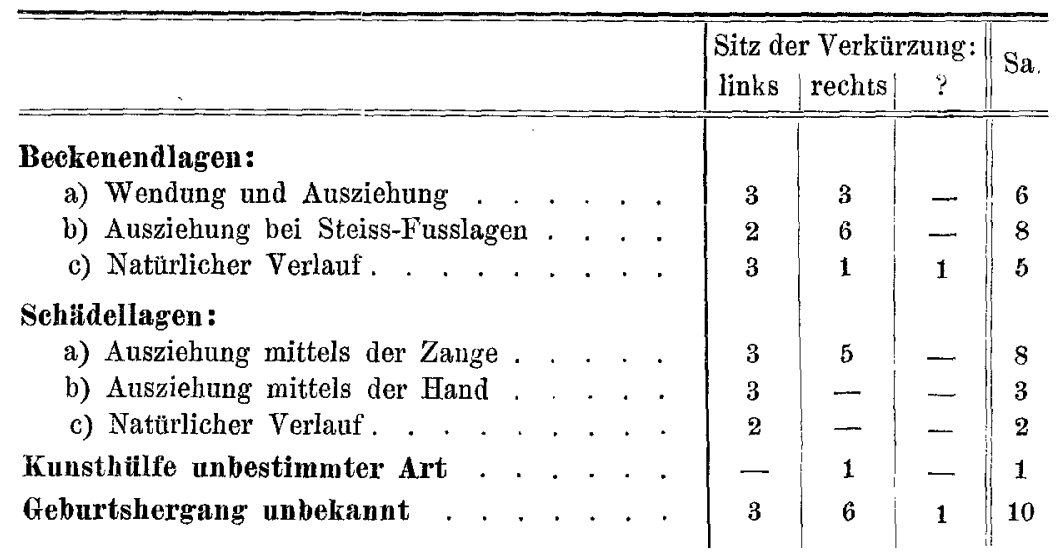


Es würde ein Leichtes gewesen sein, aus der Literatur noch eine mindestens ebenso grosse Zahl von Fällen zusammenzustellen, in welchen die schiefhalsigen Kinder in Beckenendlage oder am Kopfe ausgezogen worden sind; dann hätten aber die Beobachtungen mit natürlichem Verlaufe gefehlt, da bisher Veröffentlichungen nicht vorhanden sind, welche das Gesammtmaterial grösserer Anstalten umfassen.

Fernerhin ist mit Stromeyer fehlerhafter Weise bisher die Gruppe der natürlich verlaufenen Steisslagen nicht von den mit Zug beendeten abgetrennt worden. Wohl mag von unseren 5 Fällen, in denen es einfach hiess: in Steisslage geboren, noch der eine und andere den Eltern unbewusst durch Ausziehung beendet sein. Unsere Hebammen und auch unsere Aerzte haben nicht selten die Ansicht, dass am herausgetretenen Beine oder Steisse überhaupt immer gezogen werden müsse. Trotzdem stellen wir, um jedem Vorwurfe zu begegnen, gleich den 2 uatürlich verlaufenen Kopflagen, den 11 durch $\mathrm{Zug}$ beendeten, so die 5 natürlich verlaufenen Beckenendlagen den 14 gegenüber, bei denen die Ausziehung vermerkt wurde. Denn nicht die Geburt in Steisslage an sich hat ursächlich etwas mit der Verletzung des Kopfnickers zu thun. Es kommt, wie bei schwierigen Zangenextractionen, noch etwas hinzu, und zwar die oft gewaltige Zerrung, welche, an dem vorangehenden Theile ausgeübt, denselben von dem noch in den Geburtswegen festgehaltenen unter starker Dehnung des Halses entfernt. - Bei Beckenendlagen geschieht das durch den Prager Handgriff und verwandte Verfahren, die nur am Rumpfe angreifen. Es lässt sich sogar im Allgemeinen der Zeitpunkt angeben, zu dem die Verletzung erfolgt. Der Kopf wird nach oben in querer oder schräger Stellung festgehalten; ein in der Richtung der Führungslinie des Beckens ausgeübter Zug dehnt besonders die hintere Halsseite und veranlasst hier den Kopfnickerriss, bei der häufigeren ersten Beckenendlage, also rechts (vergl. A. I, b) ; die Wendung bringt den Rücken wohl ebenso häufig nach links als nach rechts, es ist also bei der Ausziehung entsprechend bald die rechte, bald die linke Halsseite stärker gedehnt (vergl. A. I, a). - Auch bei der gewaltsam beendeten Geburt in Kopflage werden die beiden grossen Theile von einander entfernt unter einer Zerrung des Halses, welche auch hier wohl gewöhnlich auf einer Seite überwiegt; quer oder schräg eingestellt könuen die oben zurückgehaltenen Schultern nicht folgen. ' Im Uebrigen ist der Mechanismus der Verletzung nicht so ohne weiteres klar. Nach unserer Statistik müsste dom Ueber- 
152 Witzel, Ueber die Entstehg. des sog. angeb. muskul. Schiefhalses.

wiegen des rechtsseitigen Kopfnickerrisses entsprechend die Zerrung besonders an der nach vorn gerichteten Halsseite erfolgen, da doch zweifelsohne die Zange häufiger an einem in erster Lage befindlichen Kopfe angelegt wird. Unsere kleinen Zahlen mögen aber auch nur einem Zufall entsprechen. Weiterhin wollen wir nicht verfehlen, darauf hinzuweisen, dass auch die Möglichkeit der Quetschung eines Muskels durch den Zangenrand besteht.

Aus dem vorliegenden ersten Versuche einer Statistik, deren Mängel uns durchaus nicht entgehen, weitere Schlüsse zu ziehen, wäre mindestens verfrüht. Immerhin tragen unsere Beobachtungen sonder Zweifel bei zur Stütze der Meinung Stromeyer's über den Zusammenhang zwischen Verletzung bei der Geburt und dem sogenannten angeborenen muskulären Schiefhalse. Verzweifelt wenig Lehren unserer Wissenschaft würden nach unserer Meinung bestehen, wenn man ein Material ähnlich dem oben erbrachten nicht als beweiskräftig erachten will. Es bleibt noch Manches dunkel in der Lehre vom congenitalen Torticollis; vor allem wird die Zukunft auch lehren müssen, ob nicht manche der schlechtweg als angeboren bezeichneten Kopfnickerverkürzungen weder vor der Geburt bestanden, noch durch diese veranlasst wurden, sondern vielmehr erst während der ersten Lebensjahre aus unbekannten Ursachen zur Ausbildung kamen.

\section{Nachtrag:}

Die vorstehenden Seiten waren schon dem Drucker übergeben als eine neue, den Gegenstand betreffende Aeusserung Peters en's im ersten Hefte des ersten Bandes der Zeitschrift für orthopädische Chirurgie erschien: Ueber don angeborenen muskulären Schiefhals. In einer für die Volkmann'sche Sammlung klinischer Vorträge bestimmten zusammenfassenden Arbeit über den muskulären Schiefhals wird der Aufsatz meines hochgeschätzten Fachgenossen von mir näher gewürdigt werden. 